\title{
MANY-BODY CORRELATIONS IN NUCLEI AND QUANTUM DOTS
}

\author{
D.J. DEAN \\ Physics Division, Oak Ridge National Laboratory, P.O. Box 2008, Bldg 6003, MS \\ 6373, Oak Ridge, TN 37831-6373, USA \\ E-mail: deandj@ornl.gov
}

\begin{abstract}
Confined quantum many-body systems of a given particle number exhibit a variety of intrinsic shape characteristics as a function of increasing external field and internal thermal excitation. The shell model is an important tool for the theoretical description of these various structures and transitions in nuclei. Another system in which correlations beyond the mean field may play an important role is semiconductor quantum dots. In this Proceedings, I will compare nuclei and quantum dots and their various deformation properties. I will report on shell-model calculations in nuclei and some recent mean-field calculations of the thermal properties of quantum dots.
\end{abstract}

\section{Phases in nuclei}

Nuclei often exhibit different intrinsic structures within the same system. Experimental and theoretical comparisons for ${ }^{56} \mathrm{Ni}$ confirmed the existence of a spherical ground-state band and a prolate deformed band beginning with a measured $2^{+}$state at $5.3 \mathrm{MeV}$ excitation energy ${ }^{1}$ (the second $0^{+}$state was experimentally inaccessible). This structure, which is present in shell-model calculations, was shown to have an intrinsic deformation by investigating the energy surface generated from cranked Hartree-Fock calculations ${ }^{2}$. This is just one example demonstrating how nuclear spectra often exhibit a band structure that is related to the intrinsic deformation in the system.

Some nuclei show interesting structure in their ground states. For example, nuclei in the ${ }^{124} \mathrm{Xe}$ region are known to be $\gamma$-soft. Detailed shell-model Monte Carlo (SMMC) calculations in this region confirmed that effective twobody interactions will give rise to such structures ${ }^{3}$. As one increases the temperature in these systems, they tend toward sphericity. This effect has also been seen in rare-earth calculations ${ }^{4,5}$. Many rare-earth systems are known to have well-deformed ground states. SMMC calculations of intrinsic shapes in these nuclei confirm this in the shell-model context by using a KumarBaranger effective interaction ${ }^{5}$.

Another interesting phenomenon occurs when one approaches shell closures in neutron-rich nuclei. For example, many calculations suggest that in ${ }^{32} \mathrm{Mg}$ the $0 f_{7 / 2}$ sub-shell begins to fill before the $s d$-shell is completely full.

maiori: submitted to World Scientific on August 30, 2001 
The large $B(E 2)$ value of ${ }^{32} \mathrm{Mg}{ }^{6}$ cannot be explained unless one allows neutrons to occupy $f p$-shell orbitals. Recent SMMC results ${ }^{7,8}$ confirm the picture that an island of inversion exists in the ${ }^{32} \mathrm{Mg}$ region ${ }^{9}$. These calculations were performed using the SMMC technique in the full $1 s-0 d-0 f-1 p$ model space. The effective shell-model interaction was derived from the realistic chargedependent Bonn potential ${ }^{10}$, with modifications to the monopole terms ${ }^{11}$ to alleviate difficulties in the saturation properties of the interaction. Since this calculation was performed in two major oscillator shells, extrapolation procedures to eliminate center-of-mass contamination of the energies were implemented for the SMMC technique. With the center-of-mass elimination and a realistic effective interaction, we were able to reproduce both groundstate masses and $B(E 2)$ values across the $s d-f p$ region, both for stable and unstable nuclei. Details are given in ${ }^{7}$.

\section{Phases in quantum dots}

I now turn to another confined quantum mechanical system that exhibits interesting many-body features. Semi-conductor quantum dots are typically formed in III-V substrates, such as GaAs. They may be laterally constructed so that their spatial dimension in the vertical dimension ( $z$ direction) is small compared to the horizontal $(x, y)$ dimensions. The GaAs may be constructed in such a way that a physical confining barrier is also included, thus giving rise to confinement in all three dimensions. Electrons may then be placed into this confined structure by conductance spectroscopy, and the system may be studied. In the following, I will briefly describe some interesting thermal effects within these systems.

\subsection{Quantum dots in magnetic fields: thermal response of broken symmetry phases}

In studies of many-body phenomena in quantum dots, experimental efforts have focused on mapping the magnetic field dependence of their ground-state structure by measuring the chemical potential via capacitance spectroscopy ${ }^{12}$. Cusps and steps in the chemical potential were found to clearly separate different ranges of magnetic fields ${ }^{12,13}$. These features were identified with phase transitions in the charge density of the quantum dot. At magnetic field strengths on the order of a few tesla, all electrons become spin-polarized initiating the maximum density droplet (MDD) phase ${ }^{12}$, in which the density is constant and homogeneous at the maximum value that can be reached in the lowest Landau level. The stability of the MDD is determined by a competi-

maiori: submitted to World Scientific on August 30, 2001 
tion among the kinetic energy, external confinement, the Coulomb repulsion between electrons, and the attraction created by the Coulomb exchange term. For increasing magnetic field, the charge-density distribution of the droplet reconstructs ${ }^{14}$ with a ring of electrons breaking off from the MDD phase. This edge reconstruction has been shown via mean-field ${ }^{15}$ and density functional theory ${ }^{16}$ calculations to result from a rotational symmetry-breaking phase transition from the MDD to a Wigner molecule or Wigner crystal phase. These calculations are in good qualitative agreement with recent experimental results where instabilities of the MDD state and other transitions in the high magnetic field region were accompanied by a redistribution of the charge density ${ }^{13}$.

In describing the ground-state and low-lying (intra-band) excitations of the $N$-electron semiconductor nanostructures, it is often sufficient to restrict consideration to the conduction band using the effective-mass approximation ${ }^{17}$. We consider the problem of $\mathrm{N}$ electrons of effective mass $m^{*}$ in a plane, $(x, y)$, confined by an external parabolic potential, $V(r)=$ $\frac{1}{2} m^{*} \omega_{0}^{2} r^{2}$, and subject to a strong magnetic field $\vec{B}=B_{0} \vec{e}_{z}$. We consider the Zeeman splitting but neglect the spin-orbit interaction. The Hamiltonian for such a system is

$$
\hat{H}=\sum_{i}\left[\frac{\left(\vec{p}-\frac{e}{c} \vec{A}\right)^{2}}{2 m^{*}}+V\left(r_{i}\right)+\frac{g^{*} \mu_{B}}{\hbar} \vec{B} \cdot \vec{S}_{i}\right]+\sum_{i<j} \frac{e^{2}}{\varepsilon\left|\vec{r}_{i}-\vec{r}_{j}\right|},
$$

where the vector potential is $\vec{A}\left(\vec{r}_{i}\right)=\left(B_{0} / 2\right)\left(-y_{i}, x_{i}, 0\right), g^{*}=0.54, \varepsilon=12.9$, $m^{*}=0.067 m_{e}$, and $\hbar \omega_{0}=3 \mathrm{meV}$.

We solve this equation at the finite-temperature Hartree-Fock level ${ }^{18}$. We use a Fock-Darwin basis expansion to solve the finite-temperature HartreeFock equations. Since we use a high $(\approx 12 \mathrm{~T})$ magnetic field, we consider only angular momentum states with the $n=0$ principal quantum number. The electrons carry spin, and so our states are labeled by $k=\left\{l_{k}, s_{k}\right\}$, where $l_{k}$ is the angular momentum projection of the $k$-th state and $s_{k}$ is the spin of that state. We found convergence using fifty states for the $N \leq 8$ systems. We also checked our zero-temperature results with other publications ${ }^{15}$ for various numbers of electrons in the dot and found satisfactory agreement.

We begin the discussion of our results by investigating the electron charge, angular momentum, and spin densities as a function of increasing temperature for the $N=6$ system at $B_{0}=12.15 \mathrm{~T}$. We show densities at representative temperatures of $3.87 \mathrm{~K}$ (the low temperature limit, $\nu=6$ ), $11.97 \mathrm{~K}$ (before the first phase transition, $\nu=6$ ), $13.65 \mathrm{~K}$ (in the second phase, $\nu=5$ ), and

maiori: submitted to World Scientific on August 30, 2001 


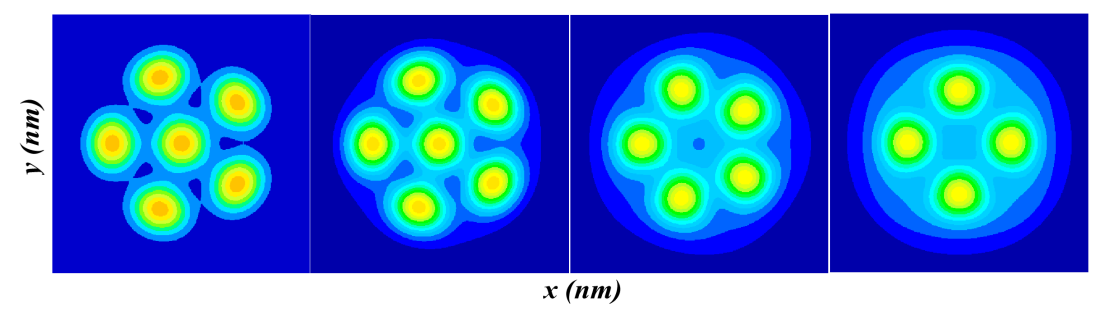

Figure 1. The charge density as a function of temperature. In each panel, $-8 \mathrm{~nm} \leq x, y \leq$ $8 \mathrm{~nm}$ and the temperature increases from left to right as discussed in the text.

$14.32 \mathrm{~K}$ (in the third phase, $\nu=4$ ), where $\nu$ is the number of definable highdensity regions (or vortices) in the charge density plots. The density, shown in Fig. 1a-d, begins as a fairly well-defined Wigner crystal at $3.87 \mathrm{~K}$, which exhibits some degree of thermal broadening at $11.97 \mathrm{~K}$. The $\nu=5$ and $\nu=4$ phases continue to show a similar amount of density in the remaining vortices, while the density of the thermally dissipated vortices have effectively spread through the entire dot.

The suddenness of the phase transitions seen in Fig. 1 become quite evident when the internal energy of the quantum dot is plotted as a function of the temperature. We show the three phases of the dot in Fig. 2a. Note that the $\nu=6$ phase exists as an excited configuration when the most probable Hartree-Fock solution is the $\nu=5$ phase. Similarly, the $\nu=4$ configuration exists as a possible excited configuration of the system even at fairly low temperatures. The specific heat, $C_{v}=d\langle H\rangle / d T$ thus shows a sharp peak structure indicating phase transitions at that the system undergoes a phase transition. This occurs since the energy is piece-wise continuous along the three phases, although some slight smoothing does occur if one includes higher-order correlations. These calculations suggest that the quantum dot exhibits a band structure of many-body levels. The low-temperature states all have the same intrinsic shape characteristic (the same vortex structure). As we increase the temperature, other $\nu$ phases become accessible. At the point when two bands of different intrinsic character cross in energy, we find a phase transition. Similar phenomena are found in nuclear physics, where at higher nuclear excitation energies the eigenstates of the system may be of a different intrinsic deformation when compared to states of the ground-state band ${ }^{19}$. 




Figure 2. Expectation value of the energy as a function of temperature showing the three phases as discussed in the text.

Signatures of the density transitions that we have seen also appear in the Hartree-Fock occupations $n_{H F}$. At low temperatures $(3.9 \mathrm{~K})$ the familiar step-function occupation behavior is evident in which the occupied HartreeFock states have $n_{H F} \approx 1$, and all other states show negligible occupation. At $12 \mathrm{~K}$ in the $\nu=6$ phase, we find a decrease of occupation to roughly 0.8 in the lowest six Hartree-Fock levels and a spreading to higher energy states. As the system undergoes the transition to $\nu=5$, we see only five Hartree-Fock levels significantly filled (with $n_{H F}>0.7$ ), and finally in the $\nu=4$ phase, only four Hartree-Fock levels are significantly filled. The occupation number-spreading, which is due to thermal excitation of the system, is enhanced significantly when the system undergoes a phase transition.

The phase transitions that we have shown in the preceding discussion have definite observable consequences. In Fig. 3 a we plot the chemical potential, that is, the separation energy $\Delta(N, T)=E_{N}(T)-E_{N-1}(T)$ to remove a particle from the quantum dot at a given temperature. Since this is an energy difference, $\Delta(N, T)$ will be influenced by transitions within both the $N=6$ and $N=5$ systems, and we expect changes in slope at the transition points. The $\nu=5$ to $\nu=4$ transition in the $N=5$ dot occurs at roughly $12 \mathrm{~K}$, causing a sharp rise in $\Delta(N=6)$. A slope change in $\Delta(N=6)$ is seen at $\approx 13 \mathrm{~K}$, where the $\nu=6$ to $\nu=5$ transition occurs in the $N=6$ system. The 
decrease from $14-14.3 \mathrm{~K}$ occurs when the $N=6$ system makes the transition from $\nu=5$ to $\nu=4$. A final change in slope occurs when the $N=5 \operatorname{dot}$ makes the transition from $\nu=4$ to $\nu=3$. For the brief temperature interval when the $N=6$ and $N=5$ dots are in the same $\nu$ phase, we see a decrease in the chemical potential.

We observe similar changes in the inverse compressibility, $\Delta_{2}(N, T)=$ $E_{N+1}(T)-2 E_{N}(T)+E_{N-1}(T)$. This quantity has been measured for quantum dots in low magnetic fields ${ }^{20}$ and studied in Hartree-Fock theory for groundstate properties ${ }^{21}$. In our case, the $N=7,6$, and 5 dots participate in the observable. We again notice strong effects as one passes through transition points in either of the three systems contributing to the observable. Figure $3 \mathrm{~b}$ shows $\Delta_{2}(N=6)$ as a function of temperature. Before transitions occur, $\Delta_{2}$ remains fairly constant. A large decrease begins at $12 \mathrm{~K}$, where the $N=5$ system undergoes its first transition. Interestingly, $\Delta_{2}$ increases significantly when the $N=6$ and $N=5$ dots are in the $\nu=4$ phase.

In order to investigate the sensitivity of our results to the approximations made (namely the Hartree-Fock approximation), we extended our studies to include the second-order perturbative correction to the Hartree-Fock energy. This correction to the energy is given by

$$
\Delta E_{2}=\frac{1}{4} \sum_{a b \leq \epsilon_{F}} \sum_{r s>\epsilon_{F}} \frac{|\langle a b|\bar{v}| r s\rangle|^{2}}{\varepsilon_{a}+\varepsilon_{b}-\varepsilon_{r}-\varepsilon_{s}}
$$

where we restrict the sums to be below and above the Fermi energy surface, $\epsilon_{F}$. In this expression, the Hartree-Fock states are given by $a, b, r, s$, with associated single-particle energies $\varepsilon_{a}, \varepsilon_{b}, \varepsilon_{r}, \varepsilon_{s}$, and $\langle a b|\bar{v}| r s\rangle$ are the antisymmetrized two-body Hartree-Fock matrix elements of the original two-body interaction of the Hamiltonian. We note that the absolute value of $\Delta E_{2}$ in our calculations is always less than $1 \%$ of the total energy, and in fact only slightly changes the observable quantities, as is evident from Fig. 3, where the second-order perturbative results are also shown for $\Delta$ and $\Delta_{2}$.

\section{Conclusion}

Confined quantum-mechanical systems exhibit a variety of interesting phenomena. Nuclear shapes range from spherical (near closed shells) to well deformed (in mid-shell systems), to those shapes with special symmetries such as the $\gamma$-soft nuclei in the ${ }^{124}$ Xe region. Quantum dots also show interesting shape changes as one adds electrons at a given magnetic-field strength and temperature. The shells that are built up in quantum dots at high magnetic fields are somewhat different in character from those found in nuclei. For

maiori: submitted to World Scientific on August 30, 2001 


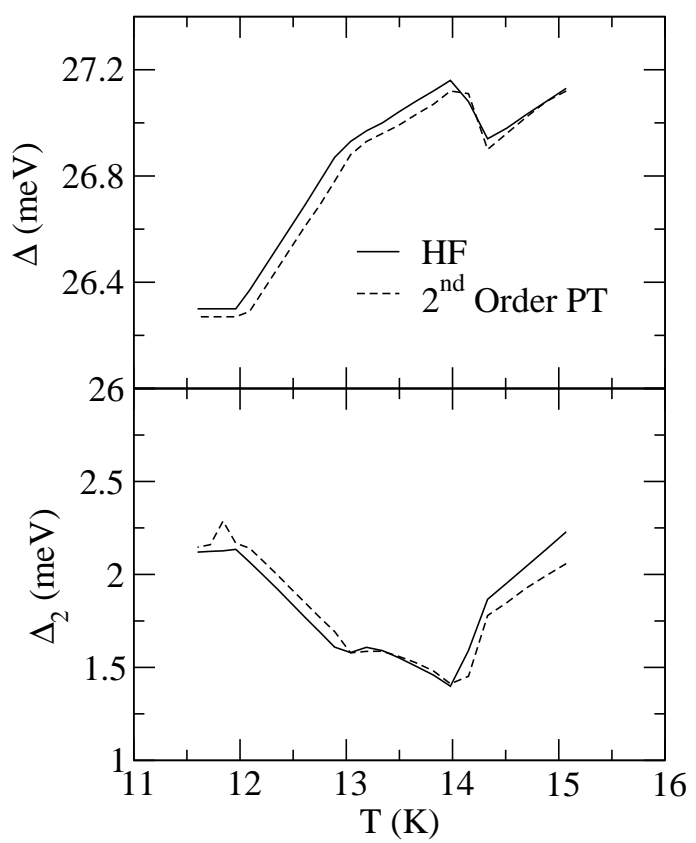

Figure 3. a) Chemical potential, $\Delta$ and b) inverse compressibility, $\Delta_{2}$ for the $N=6$ system as a function of the temperature. Hartree-Fock results are shown as a solid line, and the second-order perturbation theory results are given by the dashed line.

example, in the oscillator basis the doubly magic nucleus, ${ }^{40} \mathrm{Ca}$, can be considered as a core, and hence inert, system in calculations of low-lying states in the mid- $f p$ shell. In contrast to this behavior, the oscillator levels are all active at high magnetic fields. From density plots and second-order groundstate energy differences, one clearly sees that the shell structure in quantum dots is actually classical ${ }^{22}$.

Nuclei exhibit shape and phase transitions as a function of cranking frequency. The signature for such a change is a level crossing in $J_{z}$ as a function of the cranking frequency. Furthermore, the same nucleus can have different bands built upon different intrinsic structures within the same nucleus. This is also true in quantum dots. I indicated how a dot goes through phase transitions as a function of temperature and how different intrinsic structures are evident during the transitions. The thermal phase transitions should be experimentally verifiable by investigating fluctuations of the chemical potential 
as a function of temperature.

\section{Acknowledgements}

This research was sponsored by the U.S. Department of Energy under Contract No. DE-AC05-00OR22725 managed by UT-Battelle, LLC. The author acknowledges useful discussions and collaborations during the course of the quantum dot work with J.C. Wells, M.R. Strayer, C. Ozen, and J. Schiffer.

\section{References}

1. D. Rudolph, et al., Phys. Rev. Lett. 82 (1999) 3763.

2. T. Mizusaki, et al., Phys. Rev. C59 (1999) R1846.

3. Y. Alhassid, et al., Phys. Rev. Lett. 77 (1996) 1444.

4. D.J. Dean, et al., Phys. Lett. B317 (1993) 275.

5. J.A. White, et al., Phys. Rev. C61 (2000) 044327.

6. T. Motobayashi et al., Phys. Lett. B346 (1995) 9.

7. D.J. Dean, et al., Phys. Rev. C59 (1999) 2474.

8. P.-G. Reinhard, et al., Phys. Rev. C60 (1999) 014316.

9. X. Campi, et al., Nucl. Phys. A251 (1975) 193.

10. M. Hjorth-Jensen, et al., Phys. Rep. 261 (1995) 125.

11. A. Abzouzi, et al., Phys. Rev. Lett. 66 (1991) 1134.

12. R. C. Ashoori, Nature 379 (1996) 413.

13. T.H. Oosterkamp, et al., Phys. Rev. Lett. 82 (1999) 2931.

14. C. Chamon, et al., Phys. Rev. B 49 (1994) 8227.

15. H.-M. Müller and S.E. Koonin, Phys. Rev. B 54 (1996) 14532.

16. S.M. Reimann, et al., Phys. Rev. Lett. 83 (1999) 3270.

17. L. Jacak, P. Hawrylak, and A. Wójs, Quantum Dots (Springer, Berlin, 1997).

18. D.J. Dean, et al., Phys. Rev. B (in press, 2001).

19. D.G. Sarantites, et al., Phys. Rev. C57 (1998) R1.

20. U. Sivan et al., Phys. Rev. Lett. 77 (1996) 1123; S.R. Patel et al., Phys. Rev. Lett. 80 (1998) 4522.

21. S. Levit and D. Orgad, Phys. Rev. B60 (1999) 5549.

22. D.J. Dean et al., to be published. 